\title{
Microlocal Inverse Image and Bimicrolocalization
}

By

Kiyoshi TAKEUCHI*

\section{Contents}

\section{$\S 1$. Introduction}

$\S 2$. Review on Preliminary Notions

\$3. Microlocal Inverse Image and a Solution to the Conjecture

$\S 4$. Applications to Edge of the Wedge Type Theorems

$\S 5$. Solvability of Systems with Multiple Characteristics

\section{§ 1. Introduction}

The theory of bimicrolocalization is a natural improvement of the so-called second microlocalization. This theory, which was first considered by Kataoka-Tose [13], was introduced fully functorially by [19] and [21]. After these algebraic constructions, the theory took its full strength with $\mathscr{D}$-modules and revieled to be useful in some kinds of problems concerning partial differential equations. Until now, we can list up three types of applications:

(a) Edge of the wedge type theorems for hyperfunction solutions ([22],[23])

(b) Extension of holomorphic solutions ([23])

(c) Solvability of systems with multiple characteristics ([24])

The aim of this paper is to extend the results $(a)$ and $(c)$ with the aid of the theory of microlocal inverse image initiated by Kashiwara-Schapira [10], D'Agnolo [3] and [4]. In particular, in [23] we proposed a conjecture on the vanishing of the cohomologies of bimicrofunction solution complexes to some class of $\mathscr{D}$-Modules. Now in this paper, we solve this conjecture to generalize

Communicated by M. Kashiwara, May 16. 1997.

AMS Classification: 32C38, 35A27, 35B60. 35G15

*Department of Mathematics, University of Hiroshıma. 1-3-1. Kagamivama, Higashi-Hıroshima. Hiroshıma 739-8526, Japan.

e-mall: takeuchı@.top2.math.sci.hıroshıma-u.ac.jp 
the results of [23] and [24]. Note that the technique of microlocal inverse image enables us to "cut off" the characteristic varieties of $\mathscr{D}$-modules and characterize the conditions of the extension of solutions in terms of the geometry of them.

\section{§ 2. Review on Preliminary Notions}

In this paper, we essentially employ the terminology of [10] and [19]. Let $X \supset L \supset M$ be a sequence of $C^{\infty}$-manifolds. Let us denote it by $(X, L, M)$ and call it a triplet of manifolds. First recall the construction of the binormal deformation of $X$ along $(L, M)$ in [19]. We shall denote it by $\bar{X}_{M L}$ and let $t, s \in$ $\mathbb{R}$ be the deformation parameters. Then we have a commutative diagram

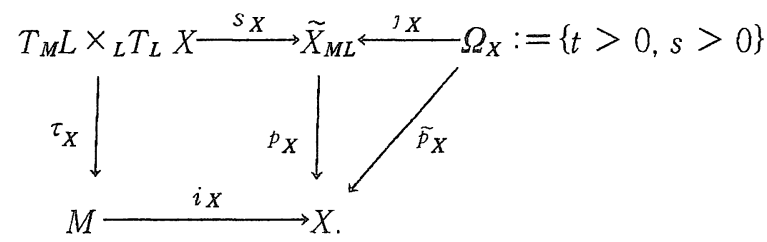

By the immersion $s_{X}$ in (2.1), $T_{M} L \times_{L} T_{L} X$ is identified with $\widetilde{X}_{M L} \cap\{t=s=0\}$. If we choose a local coordinate system $x=\left(x^{\prime}, x^{\prime \prime}, x^{\prime \prime \prime}\right)$ of $X$ such that

$$
\left\{\begin{array}{l}
L=\left\{x^{\prime}=0\right\} \\
M=\left\{x^{\prime}=0, x^{\prime \prime}=0\right\}
\end{array}\right.
$$

then the morphism $p_{X}$ in (2.1) is described by:

$$
\left(x^{\prime}, x^{\prime \prime}, x^{\prime \prime \prime}, t, s\right) \longmapsto\left(t s x^{\prime}, t x^{\prime \prime}, x^{\prime \prime \prime}\right) \text {. }
$$

We call such a coordinate system "admissible". Let $\mathbb{D}^{b}(*)$ be the derived category of $\mathbb{C}$-vector spaces on a topological space with bounded cohomologies. In [19] we defined the functor of bispecialization :

$$
\nu_{M L}: \mathbb{D}^{\mathrm{b}}(X) \longrightarrow \mathbb{D}^{\mathrm{b}}\left(T_{M} L \times{ }_{L} T_{L} X\right)
$$

by the formula :

$$
\nu_{M L}(F):=s_{X}^{-1} R j_{X *} \widetilde{p}^{-1} F
$$

We also defined two functors :

$$
\left\{\begin{array}{l}
\nu \mu_{M L}: \mathbb{D}^{\mathrm{b}}(X) \longrightarrow \mathbb{D}^{\mathrm{b}}\left(T_{M} L \times{ }_{L} T_{L}^{*} X\right) \\
\mu_{M L}: \mathbb{D}^{\mathrm{b}}(X) \longrightarrow \mathbb{D}^{\mathrm{b}}\left(T_{M}^{*} L \times{ }_{L} T_{L}^{*} X\right)
\end{array}\right.
$$

as the Fourier-Sato transformations of $\nu_{M L}(*)$. For $F \in \mathbb{D}^{\mathrm{b}}(X), \nu_{M L}(F)$, $\nu \mu_{M L}(F)$ and $\mu_{M L}(F)$ are biconic objects, that is, every cohomology sheaf of them is locally constant along two $\mathbb{R}_{+}$-orbits. 
First we shall recall the estimation of the support of the complex $\mu_{M L}(F)$ for $F \in \mathbb{D}^{\mathrm{b}}(X)$.

Theorem 2.1 (Funakoshi $[6 !$ and $[24])$. For $F \in \mathbb{D}^{\mathrm{b}}(X)$, we have :

$$
\operatorname{supp} \mu_{M L}(F) \subset T_{M_{M} \times T_{L} T_{L} X}\left(T_{L}^{*} X\right) \cap C_{T_{L}^{* X}}(S S(F)) \text {, }
$$

where we used the natural isomorphism $\left.T_{M}^{*} L \times{ }_{L} T_{L}^{*} X \simeq T_{(M \times}^{*}{ }_{L} T_{L}^{*} X\right)\left(T_{L}^{*} X\right)$ and the Hamiltonian isomorphism $-H: T^{*}\left(T_{L}^{*} X\right) \simeq T_{\left(T_{L}^{*} X\right)}\left(T^{*} X\right)$.

Let $f: M \rightarrow M^{\prime \prime}$ be a smooth morphism of real analytic manifolds, $N \subset M$ a submanifold of codimension $d \geq 1$ such that $g=f_{\mid}: N \rightarrow M^{\prime \prime}$ is smooth, and $f_{\mathbf{C}}$ : $X \rightarrow X^{\prime \prime}$ (resp. $\left.g_{\mathrm{C}}: Y \rightarrow X^{\prime \prime}\right)$ a complexification of $f\left(\right.$ resp. $g$ ). Set $L:=f_{\mathrm{C}^{1}}\left(M^{\prime \prime}\right)$ (resp. $\left.H:=g \overline{\mathbf{c}}^{1}\left(M^{\prime \prime}\right)\right)$ in $X$ (resp. in $Y$ ). We assume that $\operatorname{dim}^{\mathbf{R}} M=n$ and $\operatorname{dim}$ ${ }^{\mathbb{R}} M^{\prime \prime}=n-l$ (hence $d \leq l$ ). Thanks to the abstract edge of the wedge theorem of Kashiwara [8], we get the definition below.

Definition 2.2 ([13], [19] and [22]). We define the sheaves of bimicrofunctions $\mathscr{C}_{N L}, \mathscr{C}_{M L}$ and $\mathscr{C}_{N H}$ by :

$$
\left\{\begin{array}{l}
\mathscr{C}_{N L}:=\mu_{N L}\left(\mathscr{O}_{X}\right) \otimes \text { or }_{N}[n] \\
\mathscr{C}_{M L}:=\mu_{M L}\left(\mathscr{O}_{X}\right) \otimes \text { or }_{M}[n] \\
\mathscr{C}_{N H}:=\mu_{N H}\left(\mathscr{O}_{Y}\right) \otimes \text { or }_{N}[n-d],
\end{array}\right.
$$

where $\mathscr{O}_{X}\left(\right.$ resp. $\left.\mathscr{O}_{Y}\right)$ denotes the sheaf of holomorphic functions on $X$ (resp. $Y$ ). Now we define the relative cotangent bundle $T^{*}\left(X / X^{\prime \prime}\right)$ by the exact sequence:

$$
0 \longrightarrow V:=X \times_{X^{\prime \prime}} T^{*} X^{\prime \prime} \longrightarrow T^{*} X \longrightarrow T^{*}\left(X / X^{\prime \prime}\right) \longrightarrow 0 .
$$

Then we have a sequence of closed embedings:

$$
T_{Y}^{*} X \longrightarrow T_{Y}^{*} X \times_{X} V \longrightarrow T^{*}\left(X / X^{\prime \prime}\right) \times_{X} V \simeq T_{V}\left(T^{*} X\right)
$$

defined by the zero-section of $V \subset T^{*} X$ and the Hamiltonian isomorphism. We denote by $\mathscr{D}_{X}$ the sheaf of ring of holomorphic differential operators on $X$. In [23], we proved the following result.

Proposition 2.3. Let $\mathcal{M}$ be a coherent $\mathscr{D}$-module whose characteristic variety satisfies the condition:

$$
\dot{T}_{Y}^{*} X \cap C_{V}(\operatorname{ChM})=\emptyset .
$$

Then we have a canonical isomorphism:

$$
\mathbf{R} \rho_{!} \mathbf{R} \mathscr{H}_{o m_{\mathscr{D}_{X}}}\left(\mathcal{M}, \mathscr{C}_{N L}\right)[d] \stackrel{\sim}{\longrightarrow} \mathscr{H}_{o m_{D_{Y}}}\left(\mathcal{M}_{Y}, \mathscr{C}_{N H}\right),
$$

where $\rho: T_{N}^{*} L \times{ }_{L} T_{L}^{*} X \rightarrow T_{N}^{*} H \times{ }_{H} T_{H}^{*} Y$ is the natural projection and $\mathcal{M}_{Y}$ denotes the 
induced system of $\mathcal{M}$ on $Y$.

Since the morphism $\rho$ is finite on the support of the complex $\mathbb{R}_{\mathcal{H}} \mathscr{H}_{\mathscr{T}_{\mathrm{X}}}\left(\mathcal{M}, \mathscr{C}_{N L}\right)$ $[d]$ under the assumption (2.11), we have:

$$
H^{\prime} \mathbb{R} \mathscr{H}_{o m_{D_{X}}}\left(\mathcal{M} . \mathscr{C}_{N L}\right)=0 \text {, for } j<d .
$$

In [23], this result has been essentially used to get several edge of the wedge type theorems, but the condition (2.11) on $\mathcal{M}$ was too strong at the zero-section $X$ of $T^{*} X$. As a final style, we proposed in [23] the following conjecture. Consider the injection :

$$
T_{Y}^{*} X \times_{X} V \longrightarrow T^{*}\left(X / X^{\prime \prime}\right) \times_{X} V \simeq T_{V}\left(T^{*} X\right)
$$

and the projection $\dot{\pi}_{Y}: \dot{T}_{Y}^{*} X \times_{X} V \longrightarrow V$, and recall the definition of Bony [1].

Defimieion 2.4 (Bony [1]). We say that the system $\mathcal{M}$ is nonmicrocharacteristic for $Y$ along $V$ at $q \in V$ if $\mathcal{M}$ satisfies $\dot{\pi}_{Y}^{-1}(q) \cap C_{V}(\mathrm{Ch} \mathcal{M})=\emptyset$. Now we have:

Conjecture ( $[23])$. Let $p \in T_{N}^{*} L \times{ }_{L} T_{L}^{*} X$ and $q \in N \times{ }_{L} T_{L}^{*} X \subset V$ be its base point. If the system $\mathcal{M}$ is non-microcharacteristic for $Y$ along $V$ at $q \in V$, then we have:

$$
H^{\prime} \mathbb{R} \mathscr{H}_{o m_{\mathscr{T}_{x}}}\left(\mathcal{M}, \mathscr{C}_{N L}\right)_{p} \simeq 0 \text { for } j<d .
$$

In the next section. we will solve this conjecture under the additional condition $\dot{T}_{Y}^{*} X \cap \mathrm{Ch} M=\emptyset(\mathcal{M}$ is non-characteristic for $Y$ ).

\section{§ 3. Microlocall Inverse Image and a Solution to the Comjecture}

First, let us recall the theory of microlocal inverse image developed by Kashiwara-Schapira [10], D'Agnolo [3] and D'Agnolo-Schapira [4]. This theory was invented to obtain deep results without using pseudo-differential operators. In fact. combined with the micro-support theory of KashiwaraSchapira [10], we can obtain many new applications on the solutions to $\mathscr{D}$-modules. For example, see [4]. Delort [5] and Tonin [25]. Now let $X$ be a vector space and $0 \in X$ its origin. We will identify the tangent space $T_{0} X$ at 0 with $X$ itself, and we take a closed convex cone $\gamma$ of $T_{0} X \simeq X$. Let $q_{1}$ and $q_{2}$ denote the first and second projection from $X \times X$ to $X$ respectively. We also consider the map $s: X \times X \longrightarrow X$ defined by $\left(x, x^{\prime}\right) \longmapsto x-x^{\prime}$ and take an open neighborhood $\omega$ of 0 in $X$. The following definition is essentially given in Proposition 6.14 of [10] (see also Definition 1.3 .1 of [4]).

Definition 3.1. For $F \in \mathbb{D}^{\mathrm{b}}(X)$, we set: 


$$
\Phi_{X}(\gamma, \omega, F):=\mathbb{R} q_{2 *}\left(s^{-1} \mathbf{C}_{\gamma} \otimes^{L} q_{1}^{-1} F_{\omega}\right) .
$$

Since $\mathbb{R} q_{2} *\left(s^{-1} \mathbf{C}_{\{0\}} \otimes{ }^{L} q_{1}^{-1} F\right) \simeq F$, we have a canonical morphism

$$
\Phi_{X}(\gamma, \omega, F) \longrightarrow F .
$$

The next proposition is the main part in the theory of microlocal inverse image.

Proposition 3.2 (Proposition 6.1.4 of [10]). Let $K$ be a proper closed convex cone of $T_{0}^{*} X$ and $U \subset \subset K$ an open cone. For $F \in \mathbb{D}^{b}(X)$, let $W$ be a conic neighborhood of $K \cap(S S F \backslash\{0\})$ in $T_{0}^{*} X$. Then there exists $F_{0} \in \mathbb{D}^{\mathrm{b}}(X)$ and a morphism $u_{0}: F_{0} \longrightarrow F$ such that:

(i) $u_{0}$ is an isomorphism on $L^{\circ} \subset T_{0}^{*} X$.

(ii) $T_{0}^{*} X \cap S S F_{0} \subset W$.

In fact, if we choose a pair $(\gamma, \omega)$ satisfying some appropriate conditions (such pairs are called "refined cutting pair" in [4]) and $K^{\circ a} \subset \gamma \subset U^{\circ a}$, then the object $F_{0}:=\Phi_{X}(\gamma, \omega, F)$ and the canonical morphism

$$
u_{0}: F_{0} \longrightarrow F
$$

satisfy the conditions of the above proposition. In order to avoid the appearance of the additional parts of $\mathrm{SSF}_{0}$ in $\partial \gamma^{\circ a} \backslash\{0\}$, we have to impose these very delicate conditions on the pair $(\gamma, \omega)$.

Now we will come back to the situation considered in the last part of Section $2\left(f: M \rightarrow M^{\prime \prime}\right.$ is a smooth morphism of real analytic manifolds etc.). We also inherit the notations there. Then we have the following theorem as an answer to the conjecture in [23].

Theorem 3.3. Let $p \in T_{N}^{*} L \times{ }_{L} T_{L}^{*} X$ and $q \in N \times{ }_{L} T_{L}^{*} X \subset V$ its base point. We assume the $\mathscr{D}_{X}$-module $\mathcal{M}$ satisfics the following conditions:

(i) $M$ is non-characteristic for $Y$.

(ii) $M$ is non-microcharacteristic for $Y$ along $V$ at $q$.

Then we have:

$$
H^{\prime} \operatorname{R} \mathscr{H} o m \Phi_{X}\left(M, \mathscr{C}_{N L}\right)_{p} \simeq 0 \text { for } j<d .
$$

Proof. Let us assume $X=\mathbb{C}^{n} \simeq \mathbb{R}^{2 n}$ and the base point of $q$ in $X$ is $0 \in \mathbf{C}^{n}$. We may also assume $q \in T_{0}^{*} X$, because the case $q \in T_{X}^{*} X$ is already proved in Proposition 4.6 of [23]. Thus we can take a proper closed convex cone $K \subset$ $T_{0}^{*} X$ and an open cone $L \subset \subset \subset$ satisfying $q \in U$. If we cut off by Proposition 3.2 the micro-support of the complex $F:=\mathbb{R}^{\prime} \mathscr{H}_{o m \mathscr{D}_{X}}\left(\mathcal{M}, \mathscr{O}_{X}\right) \in \mathbb{D}^{\mathrm{b}}(X)$ with respect to this choice of cones $U \subset \subset K$, we get $F_{0} \in \mathbb{D}^{\mathrm{b}}(X)$ and a morphism $u_{0}$ : $F_{0} \longrightarrow F$ such that:
(A) $u_{0}$ is an isomorphism on $U \subset T_{0}^{*} X$.
(B) There exist an open neighborhood $W_{0}$ (not necessary conic) of $q$ in $V$ 
and an open conic neighborhood $W_{1}$ of $\{0\} \times{ }_{X} \dot{T}_{Y}^{*} X$ in $T^{*} X$ such that $\left(W_{0}+W_{1}\right) \cap S S F_{0}=\varnothing$.

This condition (B) is strictly weaker than the following one.

(C) $\dot{T}_{Y}^{*} X \cap C_{V}\left(\mathrm{SS} F_{0}\right)=\emptyset$.

As is clear from the proof of Proposition 4.6 of [23], the following isomorphism holds for the objects $F_{0} \in \mathbb{T}^{\mathrm{b}}(X)$ satisfying the condition (C):

$$
\mathbb{R} \rho \cdot \mu_{N L}\left(F_{0}\right)[2 d] \stackrel{\sim}{\longrightarrow} \mu_{N H}\left(h^{-1} F_{0}\right),
$$

where $\rho: T_{N}^{*} L \times{ }_{L} T_{L}^{*} X \rightarrow T_{N}^{*} H \times{ }_{H} T_{H}^{*} Y$ is the projection as before and $h: Y \rightarrow X$ is the inclusion. We want to use such an isomorphism, but it is difficult to get the cut-off $F_{0} \in \mathbb{D}^{\mathrm{b}}(X)$ satisfying the condition (C). This difficulty arises from the fact that the microlocal cut off works accurately only on the fiber $T_{0}^{*} X$ of $\{0\} \in$ $X$. To make up for the conditions of $F_{0} \in \mathbb{D}^{b}(X)$ we will give a precise version of Proposition 4.6 of [23]. By using the morphisms:

$$
T^{*} Y \underset{\rho_{Y}}{\stackrel{\sim}{\longrightarrow}} Y \times_{X} T^{*} X \underset{\sigma_{Y}}{\longrightarrow} T^{*} X
$$

we consider the point $q^{\prime}:=\rho_{Y} \varpi_{Y}^{-1}(q) \in N \times_{H} \dot{T}_{H}^{*} Y \subset \dot{T}^{*} Y$.

Proposidion 3.4. Let $F_{0} \in \mathbb{P}^{\mathrm{b}}(X)$ be an object which satisfies the condition (B) above at $q \in N \times{ }_{L} \dot{T}_{L}^{*} X \subset V$. Then we have an isomorphism:

$$
\mathbb{R} \rho, \mu_{N L}\left(F_{0}\right)[2 d] \stackrel{\sim}{\longrightarrow} \mu_{N H}\left(h^{-1} F_{0}\right)
$$

on $\pi_{H}^{-1}\left(q^{\prime}\right)$, where $\pi_{H}$ denotes the projection $T_{N}^{*} H \times_{H} T_{H}^{*} Y \longrightarrow N \times_{H} T_{H}^{*} Y$.

Proof. The problem being local, we may assume:

$$
\left\{\begin{array}{l}
X=\mathbb{C}^{l} \times \mathbb{C}^{n-l} \supset L=\mathbb{C}^{l} \times \mathbb{R}^{n-l} \supset M=\mathbb{R}^{l} \times \mathbb{R}^{n-l}, \\
Y=\left(\{0\} \times \mathbb{C}^{l-d}\right) \times \mathbb{C}^{n-l} \supset H=\mathbb{C}^{l-d} \times \mathbb{R}^{n-l} \supset N=\mathbb{R}^{l-d} \times \mathbb{R}^{n-l}
\end{array}\right.
$$

for $0 \leq d \leq l$. We introduce a local coordinate system $(x, y)=\left(\left(x^{\prime}, x^{\prime \prime}\right),\left(y^{\prime}, y^{\prime \prime}\right)\right), x^{\prime}$ $=\left(x_{1}, \cdots, x_{d}\right), x^{\prime \prime}=\left(x_{d+1}, \cdots, x_{n}\right), y^{\prime}=\left(y_{1}, \cdots, y_{l}\right), y^{\prime \prime}=\left(v_{l+1}, \cdots, y_{n}\right)$ of $X$ such that:

$$
\left\{\begin{array}{l}
M=\{y=0\} \supset N=\left\{y=0, x^{\prime}=0\right\} \\
L=\left\{y^{\prime \prime}=0\right\}
\end{array}\right.
$$

Take care that the numbers of $x^{\prime}$ and $y^{\prime}$-variables are different from each other. We also use the notation $y^{\prime}=\left(y_{1}, \cdots, y_{d}, y^{*}\right), y^{*}=\left(y_{d+1}, \cdots, y_{l}\right)$. In this setting, the associated admissible coordinate system of the triplet $(X, L, N)$ (resp. $(Y, H$, $N))$ is $\left(y^{\prime \prime},\left(x^{\prime}, y^{\prime}\right), x^{\prime \prime}\right)\left(\right.$ resp. $\left.\left(y^{\prime \prime}, y^{*}, x^{\prime \prime}\right)\right)$ and there exists a canonical injection $\tilde{h}: \widetilde{Y}_{N H} \rightarrow \widetilde{X}_{N L}$ induced by $h: Y \rightarrow X$. This injection is described by the equation $x^{\prime}$ $=0, y_{1}=\cdots=y_{d}=0$ and we have the commutative diagram : 


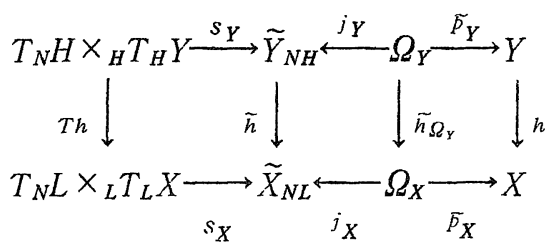

in which all squares are Cartesian. Now we take a point $p_{0}=\left(0 ; \dot{\eta}^{*}, \dot{\eta}^{\prime \prime}\right) \in$ $\pi_{H}^{-1}\left(q^{\prime}\right) \subset T_{N}^{*} H \times_{H} \dot{T}_{H}^{*} Y,\left(\dot{\eta}^{\prime \prime} \neq 0\right)$ and we will show that the canonical morphism:

$$
\mathbf{R} \rho: \mu_{N L}\left(F_{0}\right)[2 d] \longrightarrow \mu_{N H}\left(h^{-1} F_{0}\right)
$$

is an isomorphism at $p_{0}$. Let us imbed $T_{N}^{*} H \times{ }_{H} T_{H}^{*} Y$ lnto the zero-section of $T^{*}\left(T_{N}^{*} H \times{ }_{H} T_{H}^{*} Y\right)$ and consider the canonical identification:

$$
T^{*}\left(T_{N}^{*} H \times{ }_{H} T_{H}^{*} Y\right) \simeq T^{*}\left(T_{N} H \times_{H} T_{H} Y\right)
$$

which follows from Proposition 5.5.1 of [10]. Then as in the same way as the proof of Theorem 6.7 .1 of [10], it is enough to show the isomorphism:

$$
s \bar{Y}^{-1}\left[\tilde{h}^{\prime} \mathbb{C}_{\tilde{X}_{N L}} \otimes \tilde{h}^{-1} G\right] \stackrel{\sim}{\longrightarrow} s_{Y}^{-1} \tilde{h}^{\prime} G
$$

for $G:=\mathbb{R}_{X_{*}} \tilde{P}_{X}^{-1} F_{0}$ in the localized category $\mathbb{D}^{\mathrm{b}}\left(T_{N} H \times{ }_{H} T_{H} Y ; p_{0}\right)$ of $\mathbb{D}^{\mathrm{b}}\left(T_{N} H \times\right.$ $\left.{ }_{H} T_{H} Y\right)$ at $p_{0}=\left(0,0,0 ; \dot{\eta}^{*} d y^{*}+\dot{\eta}^{\prime \prime} d y^{\prime \prime}+0 d x^{\prime \prime}\right) \in T^{*}\left(T_{N} H \times_{H} T_{H} Y\right)$. Thanks to Corollary 6.4 .4 of [10] it remains to prove the following assertion.

Lemma 3.5. The natural injection $\tilde{h}: \widetilde{Y}_{N H} \rightarrow \widetilde{X}_{N L}$ is non-characteristic for the object $G=\mathbb{R}_{X_{*}} \widetilde{p}_{\bar{X}}^{-1} F_{0}$ on $V_{\varepsilon}$ for sufficiently small $\varepsilon>0$. Here the open subsets $V_{\varepsilon} \subset$ $T^{*} \widetilde{Y}_{N H}$ is defined by

$$
\begin{aligned}
V_{\varepsilon}:= & \left\{\left(y^{\prime \prime}, y^{*}, x^{\prime \prime}, t, s ; \eta^{\prime \prime}, \eta^{*}, \xi^{\prime \prime}, \tau, \sigma\right) \in T^{*} \widetilde{Y}_{N H} ;\right. \\
& \left.\left|\frac{\eta^{\prime \prime}}{\left|\eta^{\prime \prime}\right|}-\frac{\dot{\eta}^{\prime \prime}}{\left|\dot{\eta}^{\prime \prime}\right|}\right|<\varepsilon,\left|\xi^{\prime \prime}\right|<\varepsilon, \quad\left|\eta^{\prime \prime}\right|,\left|y^{\prime \prime}\right|,\left|y^{*}\right|,\left|x^{\prime \prime}\right|,|t|,|s|<\varepsilon\right\} .
\end{aligned}
$$

Proof. We will prove this lemma by a contradiction. Assume that for every $\varepsilon>0$ there exists a point $p_{\varepsilon}:=\left(y^{\prime \prime}, y^{*}, x^{\prime \prime}, t, s ; \eta^{\prime \prime}, \eta^{*}, \xi^{\prime \prime}, \tau, \sigma\right) \in V_{\varepsilon}$ at which the morphism $\tilde{h}: \widetilde{Y}_{N H} \longrightarrow \widetilde{X}_{N L}$ is characteristic for $\mathrm{SS}(G)$. We show that it contradicts the condition (B) of $S S F_{0}$. By Proposition 6.2.4 (iii) of [10] we can find the sequences :

$$
\left\{\begin{array}{l}
\left(y_{m}^{\prime \prime},\left(x_{m}^{\prime}, y_{m}^{\prime}\right), x_{m}^{\prime \prime}, t_{m}, s_{m} ; \eta_{m}^{\prime \prime},\left(\xi_{m}^{\prime}, \eta_{m}^{\prime}\right), \xi_{m}^{\prime \prime}, \tau_{m}, \sigma_{m}\right) \in \mathrm{SS}(G) \\
\left(\bar{y}_{m}^{\prime \prime}, \bar{y}_{m}^{*}, \bar{x}_{m}^{\prime \prime}, \bar{t}_{m}, \bar{s}_{m}\right) \in \widetilde{Y}_{N H}
\end{array}\right.
$$

such that

$$
\left\{\begin{array}{l}
\left(\bar{y}_{m}^{\prime \prime}, \bar{y}_{m}^{*}, \bar{x}_{m}^{\prime \prime}, \tau_{m}, \bar{s}_{m}\right) \longrightarrow\left(y^{\prime \prime}, y^{*}, x^{\prime \prime}, t, s\right) \\
\left(y_{m}^{\prime \prime},\left(x_{m}^{\prime}, y_{m}^{\prime}\right), x_{m}^{\prime \prime}, t_{m}, s_{m}\right) \longrightarrow\left(y^{\prime \prime},\left(0,\left(0, y^{*}\right)\right), x^{\prime \prime}, t, s\right) \\
\left(\eta_{m}^{\prime \prime}, \eta_{m}^{*}, \xi_{m}^{\prime \prime}, \tau_{m}, \sigma_{m}\right) \longrightarrow\left(\eta^{\prime \prime}, \eta^{*}, \xi^{\prime \prime}, \tau, \sigma\right) \cdots \mathbf{A} \\
\left|\left(\eta_{m}^{\prime \prime},\left(\xi_{m}^{\prime}, \eta_{m}^{\prime}\right), \xi_{m}^{\prime \prime}, \tau_{m}, \sigma_{m}\right)\right| \longrightarrow+\infty \quad \cdots \mathbf{B}
\end{array}\right.
$$


Note that from $\mathbb{A}$ and $\mathbb{B}$ we have

$$
\left|\left(\xi_{m}^{\prime}, \eta_{1 m}, \cdots, \eta_{d m}\right)\right| \longrightarrow+\infty .
$$

Recall that $\operatorname{SS}(G) \subset \operatorname{SS}\left(\tilde{p}_{\bar{x}}^{-1} F_{0}\right) \hat{+} N^{*}\left(\Omega_{X}\right)$ and we have by Proposition 5.4 .5 of [10]

$$
\begin{aligned}
\operatorname{SS}\left(\tilde{p}_{x}^{-1} F_{0}\right)= & \left\{\left(\frac{y^{\prime \prime}}{t s}, \frac{\left(x^{\prime}, y^{\prime}\right)}{t}, x^{\prime \prime}, t, s ; t s \eta^{\prime \prime} d y^{\prime \prime}+t \xi^{\prime} d x^{\prime}+t \eta^{\prime} d y^{\prime}+\xi^{\prime \prime} d x^{\prime \prime}+\cdots\right) ;\right. \\
& \left.\left(y^{\prime \prime},\left(x^{\prime}, y^{\prime}\right), x^{\prime \prime} ; \eta^{\prime \prime},\left(\xi^{\prime}, \eta^{\prime}\right), \xi^{\prime \prime}\right) \in \operatorname{SS}\left(F_{0}\right)\right\} .
\end{aligned}
$$

Therefore by Remark 6.2 .8 (ii) of [10], we obtain the sequences

$$
\left\{\begin{array}{l}
p_{n}=\left(y_{m,}^{\prime \prime},\left(x_{m}^{\prime}, y_{m}^{\prime}\right), x_{m}^{\prime \prime} ; \eta_{m}^{\prime \prime},\left(\xi_{m}^{\prime}, \eta_{m}^{\prime}\right), \xi_{m 2}^{\prime \prime}\right) \in \mathrm{SS}\left(F_{0}\right) \\
t_{m}, s_{m}>0 \text { and }\left(\bar{y}_{m}^{\prime \prime}, \bar{y}_{m}^{*}, \bar{x}_{m}^{\prime \prime}, t_{m}, \bar{s}_{m}\right) \in \widetilde{Y}_{N H}
\end{array}\right.
$$

such that

$$
\left\{\begin{array}{l}
\left(\bar{y}_{m}^{\prime \prime}, \bar{y}_{m}^{*}, \bar{x}_{m}^{\prime \prime}, t_{m}, \bar{s}_{m}\right) \longrightarrow\left(y^{\prime \prime}, y^{*}, x^{\prime \prime}, t, s\right) \\
\left(y_{m}^{\prime \prime} /\left(t_{m} s_{m}\right),\left(x_{m}^{\prime}, y_{m}^{\prime}\right) / t_{m}, x_{m}^{\prime \prime}, t_{m}, s_{m}\right) \longrightarrow\left(y^{\prime \prime},\left(0,\left(0, y^{*}\right)\right), x^{\prime \prime}, t, s\right) \\
\left(t_{m} s_{m} \eta_{m}^{\prime \prime}, t_{m} \eta_{m}^{*}, \xi_{m}^{\prime \prime}\right) \longrightarrow\left(\eta^{\prime \prime}, \eta^{*}, \xi^{\prime \prime}\right) \cdots \mathbb{C} \\
\left|\left(t_{m} \xi_{m}^{\prime}, t_{m}\left(\eta_{1 m}, \cdots, \eta_{d m}\right)\right)\right| \longrightarrow+\infty \quad \cdots \mathbb{D}
\end{array}\right.
$$

Set $\zeta_{k m}=\xi_{k m}+i \eta_{k m}$ for $k=1, \cdots, n$ and consider the ratio of $\left(\zeta_{1 m}, \cdots, \zeta_{d m}\right) \in \mathbb{C}^{d}$ and $\left(\zeta_{d+1, m}, \cdots, \zeta_{l m}\right) \in \mathbb{C}^{l-d}$. Then it follows from $\mathbb{C}$ and $\mathbb{D}$ we get the convergence:

$$
\left|\left(\zeta_{d+1, m}, \cdots, \zeta_{l m}\right)\right| /\left|\left(\zeta_{1 n l}, \cdots, \zeta_{d m}\right)\right| \longrightarrow 0
$$

Moreover by $\mathbb{C}$ and (3.12) we see that the directions of the vectors $\left(\zeta_{l+1, m}, \cdots\right.$, $\left.\zeta_{n m}\right) \in \mathbb{C}^{n-l}$ tend to that of a point in $W_{0} \subset V$. It contradicts the condition (B) on the micro-support of $F_{0} \in \mathbb{D}^{\mathrm{b}}(X)$.

Now let us continue the proof of the theorem. Note that by the assumption (1) of the theorem the set $\varpi_{Y} \rho_{Y}^{-1}\left(q^{\prime}\right) \cap \mathrm{SS} F$ is a finite set, and we will denote it by $\left\{q_{0}\right.$ $\left.=q, q_{1}, \cdots, q_{m}\right\} \subset T_{0}^{*} X$. Hence we can take cones $U_{l} \subset \subset K_{\imath}(i=1,2, \cdots, m)$ in $T_{0}^{*} X$ so that $q_{i} \in U_{1}$ and $K_{1} \cap K_{j}=\varnothing$ for $i \neq j$ and cut off the micro-support of $F$. We denote the corresponding cut off morphisms by $u_{\imath}: F \rightarrow F(i=1,2, \cdots, m)$. Then the morphism:

$$
\bigoplus_{l=0}^{m} h^{-1}\left(u_{\imath}\right): \bigoplus_{\imath=0}^{m} h^{-1} F_{\imath} \longrightarrow h^{-1} F
$$

defined by these microlocal cut off morphisms is an isomorphism in $\mathbb{D}^{b}\left(Y, q^{\prime}\right)$ and hence induces an isomorphism:

$$
\bigoplus_{i=0}^{m} \mu_{N H}\left(h^{-1} F_{\imath}\right) \stackrel{\sim}{\longrightarrow} \mu_{N H}\left(h^{-1} F\right)
$$


at $q^{\prime} \in N \times{ }_{H} \dot{T}_{H}^{*} Y \subset T^{*} Y$ by Theorem 2.1. By virtue of the same theorem, we get a chain of isomorphisms at $\rho(p) \in T_{N}^{*} H \times{ }_{H} \dot{T}_{H}^{*} Y$ :

$$
\begin{aligned}
& \mathbf{R} \rho \cdot \mathbf{R} \mathscr{H}_{\mathscr{D}_{X}}\left(\mathcal{M}, \mathscr{C}_{N L}\right)[d]=\mathbb{R} \rho \cdot \mu_{N L}(F) \otimes \text { or }_{N}[n+d] \\
& \stackrel{\sim}{\sim} \rho \cdot \mu_{N L}\left(F_{0}\right) \otimes \operatorname{or}_{N}[n+d] \\
& \simeq \mu_{N H}\left(h^{-1} F_{0}\right) \otimes \operatorname{or}_{N}[n-d]
\end{aligned}
$$

and the last term is a direct summand of the complex $\mu_{N H}\left(h^{-1} F\right) \otimes$ or $_{N}[n-d]=$ $\mathbb{R} \mathscr{H}_{o m_{\mathscr{D}_{Y}}}\left(\mathcal{M}_{Y}, \mathscr{C}_{N H}\right)$ by the formula (3.21). It implies

$$
H^{j}\left[\mathbb{R} \rho, \mathbb{R} \mathscr{H}_{o m_{\mathscr{D}_{x}}}\left(\mathcal{M}, \mathscr{C}_{N L}\right)\right]_{\rho(p)} \simeq 0 \text { for } j<d
$$

and by the assumption (11) of the theorem we get:

$$
H^{\prime} \mathbf{R} \mathscr{H}_{o m_{\mathscr{D}_{\lambda}}}\left(\mathcal{M}, \mathscr{C}_{N L}\right)_{p} \simeq 0 \text { for } j<d .
$$

Note that the assumption (ii) entails the finiteness of the morphism $\rho$ on the support of the complex $\mathbb{R} \mathscr{H}_{o m} \mathscr{\mathscr { D }}_{X}\left(\mathcal{M}, \mathscr{C}_{N L}\right)$. It completes the proof.

Remark 3.6. We conjecture that the condition (i) of Theorem 3.3 can be removed. In fact, under the condition (ii) we wanted to prove the isomorphism:

$$
\mathbb{R} \rho, \mathbf{R} \mathscr{H}_{o m_{\mathscr{D}_{X}}}\left(\mathcal{M}, \mathscr{C}_{N L}\right)[d] \stackrel{\sim}{\longrightarrow} \mathscr{R H}_{o m_{Y} Y}\left(\mathcal{M}_{Y}^{U}, \mathscr{C}_{N H}\right),
$$

where $U$ is an open neighborhood of $q \in V$ in $T^{*} X$ and $\mathcal{M}_{Y}^{U}$ denotes the induced system of $\left.\mathcal{M}\right|_{U}$ to $Y$ in the category of $\mathscr{E}_{X}$-modules. If one can prove this isomorphism, our conjecture is true.

\section{$\S 4$. Applications to $\mathbb{E}$ dge of the Wedge Type Theorems}

In this section, we will give some applications of Theorem 3.3 and the results in [23] to the extension of hyperfunction (real analytic) solutions. The main problem that we consider here is to find good sufficient conditions on $\mathscr{D}$-modules $\mathcal{M}$ for the vanishing of cohomologies:

$$
H^{j} \mu_{N} \mathbb{R} \mathscr{H}_{o m_{\mathscr{D}_{\lambda}}}\left(\mathcal{M}, \mathscr{B}_{M}\right) \simeq 0 \text { for } j<d,
$$

where $d$ is the codimension of $N$ in $M$. As was shown in [23] and [28], the solutions to this problem give rise to many nice applications to the extension of solutions defined on the tuboids in $M$ having $N$ as its edge. First recall a definition of [23].

Definition 4.1. We define a biconic sheaf $\mathscr{C}_{N M}$ on $T_{N}^{*} M \times_{M} T_{M}^{*} X$ by:

$$
\mathscr{C}_{N M}:=\mu_{N M}\left(\mathscr{O}_{X}\right) \otimes \text { or }_{N}[n] \text {. }
$$

As we shall see in the following proposition, the sheaf $\mathscr{C}_{N M}$ defined above 
enables us to extend the formulation of microlocal boundary value problems due to Kataoka [11] to the cases where the codimension $d$ of the boundary $N$ is greater than one.

Proposition 4.2 (Proposition 3.4 of [23]). There exists a distinguished triangle in $\mathbb{D}^{\mathrm{b}}\left(T_{N}^{*} M\right)$ :

$$
\begin{aligned}
& \mathbb{R} \theta, \mathbb{R} \mathscr{H} o m_{\mathscr{D}_{X}}\left(\mathcal{M}, \mathscr{C}_{N \mid X}\right) \longrightarrow \mu_{N} \mathbb{R} \mathscr{H}_{o m_{\mathscr{D}_{X}}}\left(\mathcal{M}, \mathscr{B}_{M}\right) \otimes o r_{N \mid M} \\
& \longrightarrow \mathbb{R} \dot{\pi}_{M}, \mathbb{R} \mathscr{H}_{o m_{\mathscr{D}_{X}}}\left(\mathcal{M}, \mathscr{C}_{N M}\right) \longrightarrow+1 \text {, }
\end{aligned}
$$

where $\theta: T_{N}^{*} X \rightarrow T_{N}^{*} M$ and $\dot{\pi}_{M}: T_{N}^{*} M \times_{M} \dot{T}_{M}^{*} X \longrightarrow T_{N}^{*} M$ are the projections.

We know by Kashiwara-Kawai's division theorem of [7] that

$$
H^{\prime} \mathbb{R} \mathscr{H}_{o m_{D_{X}}}\left(\mathcal{M}, \mathscr{C}_{N \mid X}\right) \simeq 0 \text { for } j<d
$$

if the $\mathscr{D}_{X}$-module $\mathcal{M}$ is non-characteristic for $Y$. Hence in view of $(4.3)$, to show the vanishing (4.1) it suffices to study the complex $\mathbb{R} \mathscr{H} o m_{\mathscr{D}_{X}}\left(\mathcal{M}, \mathscr{C}_{N M}\right)$ on $T_{N}^{*} M \times{ }_{M} T_{M}^{*} X$. If moreover the system $\mathcal{M}$ is elliptic, this complex vanishes there by Theorem 2.1 and one gets (4.1), which recovers the main theorem of [7]. Our formulation enables us to investigate non-elliptic $\mathscr{D}_{X}$-modules. To recall the notion of partially elliptic systems of Bony-Schapira [2], consider the relative cotangent bundle $T^{*}\left(M / M^{\prime \prime}\right)$ defined by the exact sequence :

$$
0 \longrightarrow \Lambda:=M \times_{M^{\prime}} T_{M^{\prime \prime}}^{*} X^{\prime \prime} \longrightarrow T_{M}^{*} X \longrightarrow T^{*}\left(M / M^{\prime \prime}\right) \longrightarrow 0 .
$$

Note that $\Lambda \simeq M \times{ }_{L} T_{L}^{*} X$ and it is a totally real submanifold of $V=X \times_{X^{\prime \prime}} T^{*} X^{\prime \prime}$ $\subset T^{*} X$. Now we have an injection:

$$
T^{*}\left(M / M^{\prime \prime}\right) \times_{M} \Lambda \longrightarrow T^{*}\left(X / X^{\prime \prime}\right) \times_{X} V \simeq T_{V}\left(T^{*} X\right)
$$

and a projection $\dot{\pi}_{\Lambda}: \dot{T}^{*}\left(M / M^{\prime \prime}\right) \times{ }_{M} \Lambda \longrightarrow \Lambda$.

Definition 4.3 (Bony-Schapira [2]). For $q \in \Lambda$, we say that the $\mathscr{D}_{X}$-module (system) $\mathcal{M}$ is partially elliptic along $V \subset T^{*} X$ at $q$ if

$$
\dot{\pi}_{\Lambda}^{-1}(q) \cap C_{V}(\operatorname{Ch} M)=\emptyset .
$$

In Proposition 4.3 of [23], we proved the next result.

Proposition 4.4. Let $p \in T_{N}^{*} M \times{ }_{L} T_{L}^{*} X$ and $q \in N \times{ }_{L} T_{L}^{*} X \subset \Lambda$ be its base point. Suppose that $\mathcal{M}$ is partially elliptic along $V$ at $q$. Then we have a natural isomorphism at $p$ :

$$
\mathbb{R} \mathscr{H}_{o m_{\Phi_{X}}}\left(\mathcal{M}, \mathscr{C}_{N M}\right) \stackrel{\sim}{\longrightarrow}{ }_{*} \mathbb{R} \rho_{0 *} \mathbb{R} \mathscr{H} o m_{\mathscr{D}_{X}}\left(\mathcal{M}, \mathscr{C}_{N L}\right),
$$

where we used the injection $\iota: T_{N}^{*} M \times{ }_{L} T_{L}^{*} X \longrightarrow T_{N}^{*} M \times{ }_{M} T_{M}^{*} X$ and the projection $\rho_{0}$ : $T_{N}^{*} L \times{ }_{L} T_{L}^{*} X \longrightarrow T_{N}^{*} M \times{ }_{L} T_{L}^{*} X$. 
Combining Proposition 4.2, Proposition 4.4 and Theorem 3.3, we obtain the following theorem which improves Theorem 4.8 of [23].

Theorem 4.5. We suppose that the system $M$ satisfies the conditions:

(i) $M$ is non-characteristic for $Y$.

(ii) $M$ is partially elliptic along $V$ on $N \times{ }_{L} \dot{T}_{L}^{*} X$, and $T_{M}^{*} X \cap C h M \subset T_{L}^{*} X$.

(iii) $\mathcal{M}$ is non-microcharacteristic for $Y$ along $V$ on $N \times{ }_{L} T_{L}^{*} X$.

Then we have the vanishing for every $i<d$ :

$$
H^{\jmath} \mu_{N} \mathbf{R} \mathscr{H}_{o m_{D_{\lambda}}}\left(M, \mathscr{B}_{M}\right) \simeq 0 .
$$

From this theorem, we can deduce local Bochner type extension theorems concerning the hyperfunction (real analytic) solutions to $\mathscr{D}$-modules by using a method in the proof of Theorem 1 of Uchida [28] (cf. Sato-Kawai-Kashiwara [17]). Here we denote by $\mathscr{A}_{M}$ the sheaf of real analytic functions on $M$. We say an open subset $\Omega_{0} \subset M$ is an open convex cone with the edge $N$ if locally, in a real analytic chart $M \simeq \mathbb{R}^{d} \times \mathbb{R}^{n-d}$ such that $N \simeq\{0\} \times \mathbb{R}^{n-d}, \Omega_{0}$ is expressed as $\Omega_{1} \times \mathbb{R}^{n-d}$ by a proper open convex cone $\Omega_{1}$ in $\mathbb{R}^{d}$.

Theorem 4.6. Suppose that $\mathcal{M}$ satisfies the conditions (i)-(iii) of Theorem 4.5.

(i) For every open convex cone $\Omega_{0}$ with the edge $N$ in $M$, every hyperfunction solution $\left.u \in \Gamma_{\Omega} \mathscr{H} o m_{\mathscr{D}_{\lambda}}\left(\mathcal{M}, \mathscr{B}_{M}\right)\right|_{N}$ to $\mathcal{M}$ on $\Omega:=M \backslash \bar{\Omega}_{0}$ automatically extends to an open neighborhood of $N$ as a hyperfunction solution to $\mathcal{M}$.

(ii) Let $U \subset T_{N} M$ be an open cone with connected fibers and $V \supset U$ its convex hull. Then we have isomorphisms:

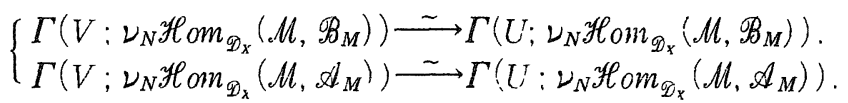

Let us mention that we have used the unique continuation property of microfunctions with holomorphic parameter to get the second isomorphism of (11). The microfunction solutions to partially elliptic systems have holomorphic parameters.

Remark 4.7. Under the same conditions as in the above theorem, we can also deduce an edge of the wedge theorem of Epstein type for hyperfunction (real analytic) solutions by using the proof of Proposition 7.2 of [28].

Example 4.8. Let $\mathcal{N}=\mathscr{D}_{X} / \sum_{j=1}^{d} \mathscr{D}_{X} Q_{j}, Q_{j} \in \mathscr{D}_{X}(j=1, \cdots, d)$ be a coherent $\mathscr{D}_{X}$-module such that:

(i) $\mathcal{N}$ is partially elliptic along $V$ on $N \times_{L} T_{L}^{*} X$ and $T_{M}^{*} X \cap \operatorname{Ch} \mathcal{N} \subset T_{L}^{*} X$

(ii) $\dot{T}_{Y}^{*} X \cap C_{V}(\mathrm{Ch} \mathcal{N})=\emptyset$

Then we have 


$$
H^{j} \mu_{N} \mathbb{R}_{\mathcal{H}} \operatorname{Hom}_{\mathscr{D}_{X}}\left(\mathcal{N}, \mathscr{B}_{M}\right) \simeq 0 \text { for } j<d
$$

The condition (ii) implies that $\mathcal{N}$ is non-microcharacteristic for $Y$ along $V$ at the zero-section of $V$. We think it is a very strong condition, because the operators $Q$,'s are a priori assumed to have symbols of the form :

$$
\sigma\left(Q_{j}\right)\left(z, \zeta^{\prime}\right)=\sum_{\left|\alpha^{\prime}\right|=m,} a_{\eta, \alpha^{\prime}}(z) \zeta^{\prime \alpha^{\prime}}, \quad z=\left(z^{\prime}, z^{\prime \prime}\right) \in \mathbb{C}^{l} \times \mathbb{C}^{n-l} \simeq X, \alpha^{\prime}=\left(\alpha_{1}, \cdots, \alpha_{l}\right)
$$

in this case. Here we denote by $z^{\prime}\left(\right.$ resp. $\left.\zeta^{\prime}\right)$ the first $l \geq d$ variables $\left(z_{1}, \cdots, z_{l}\right)$ (resp. $\left(\zeta_{1}, \cdots, \zeta_{l}\right)$ ) and $Y=\left\{z_{1}=\cdots=z_{d}=0\right\} \subset X$ (This notation is different from that in the proof of Theorem 3.3). Now thanks to Theorem 4.5 and 4.6, we know that the elliptic factors in the characteristic variety of the system are harmless when one wants to obtain edge of the wedge type theorems. For example, take elliptic operators $E_{j}(j=1, \cdots, d)$ on $X$ and set $P_{j}=E_{j} Q_{j}+$ (lower order terms). Then the system $\mathcal{M}=\mathscr{D}_{X} / \sum_{j=1}^{d} \mathscr{D}_{X} P$, does not satisfy the condition $T_{Y}^{*} X \cap C_{V}(\mathrm{Ch} \mathcal{N})=\emptyset$ but satisfies the condition (ii) and (iii) of Theorem 4.6. Therefore if the system $\mathcal{M}$ is non-characteristic for $Y$ in addition, every hyperfunction (real analytic) solution of $\mathcal{M}$ on the open tuboid $U \subset T_{N} M$ along $N$ extends to its convex hull $V \subset T_{N} M$ as a solution :

$$
\left\{\begin{array}{l}
\Gamma\left(V: \nu_{N} \mathscr{H}_{o m_{\mathscr{D}_{X}}}\left(\mathcal{M}, \mathscr{B}_{M}\right)\right) \longrightarrow \Gamma\left(U ; \nu_{N} \mathscr{H} o m_{\mathscr{D}_{x}}\left(\mathcal{M}, \mathscr{B}_{M}\right)\right) . \\
\Gamma\left(V ; \nu_{N} \mathscr{H}_{\mathscr{D}_{X}}\left(\mathcal{M}, \mathscr{A}_{M}\right)\right) \longrightarrow \Gamma\left(L^{\top} ; \nu_{N} \mathscr{H}_{\mathscr{D}_{X}}\left(\mathscr{M}, \mathscr{A}_{M}\right)\right) .
\end{array}\right.
$$

Note that the lower order terms of the operators $P_{j}$ 's could not be taken arbitrarily before we prove Theorem 4.6. See Example 5.2 of the previous paper [23]. Moreover the conditions of [23] were not totally geometric. Our conditions in Theorem 4.6 are characterized only by the geometry of the characteristic variety of the $\mathscr{D}_{X}$-modules. Notice that the $\mathscr{D}_{X}$-modules $\mathcal{M}$ obtained in this way contain partially elliptic factors and elliptic ones at the same time.

If we restrict ourselves to get the vanishing:

$$
H^{j} \mu_{N} \mathbb{R} \mathscr{H} o m_{\mathscr{D}_{X}}\left(\mathcal{N}, \mathscr{B}_{M}\right)_{\xi} \simeq 0 \text { for } j<d,
$$

for a fixed covector $\xi \in \dot{T}_{N}^{*} M$, we can further weaken the hypotheses on the $\mathscr{D}$-modules $\mathcal{M}$. In fact, we have by Theorem 2.1 the following result. We shall use the projections:

$$
T_{N}^{*} M \stackrel{\pi_{M}}{\stackrel{\pi}{*}} T_{N}^{*} M \times_{M} T_{M}^{*} X \stackrel{\pi_{N}}{\longrightarrow} N \times{ }_{M} T_{M}^{*} X .
$$

Theorem 4.9. Let $p \in \dot{T}_{N}^{*} M \times{ }_{M} T_{M}^{*} X$ and suppose that $\mathcal{M}$ is micro-hyperbolic (in the sense of [9]) in the direction $\pi_{M}(p) \in \dot{T}_{N}^{*} M \subset \dot{T}^{*} M$ at $\pi_{N}(p) \in T_{M}^{*} X$. Then we have: 


$$
\mathbf{R} \mathscr{H}_{o m_{\Phi_{X}}}\left(\mathcal{M}, \mathscr{C}_{N M}\right)_{p} \simeq 0 \text {. }
$$

Now assume that $N$ is of codimension $d \geq 2$ in $M$ and let $\xi \in \dot{T}_{N}^{*} M$ be a fixed covector. If the system $\mathcal{M}$ is hyperbolic in this direction $\xi \in \dot{T}^{*} M$, then we have $\mu_{N} \mathbf{R} \mathscr{H}_{o m_{\mathscr{C}_{x}}}\left(\mathcal{M}, \mathscr{B}_{M}\right)_{\xi} \simeq 0$ by a result of Kashiwara-Schapira [9]. On the other hand, if $\mathcal{M}$ is non-characteristic for $Y$ and elliptic, then it was shown by

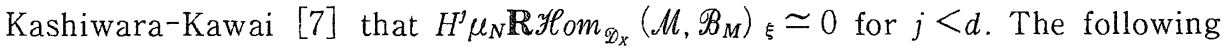
theorem is a natural generalization of these well-known facts.

Theorem 4.10. Let $\mathcal{M}$ be a coherent $\mathscr{D}$-module and $C h \mathbb{M}=\bigcup_{j=1}^{k} V$, be the irreducible decomposition of the characteristic variety of $M$. We assume that each $V$, satisfies the condition (i) or (ii) below.

(i) $V$, is non-characteristic for $Y$ and elliptic i.e., $V, \cap C h M=\emptyset$.

(ii) $V$, is hyperbolic in the direction $\xi \in T_{N}^{*} M$.

Then we have

$$
H^{j} \mu_{N} \mathbf{R} \mathscr{H}_{o m_{\mathscr{C}_{x}}}\left(\mathcal{M} . \mathscr{B}_{M}\right)_{\xi} \simeq 0 \text { for } j<d .
$$

Proof. It follows from Proposition 4.2 and Theorem 4.9 that

$$
\text { (4.15) } \mathbb{R} \theta \cdot \mathbb{R} \mathscr{H}_{o m_{\mathscr{D}_{\mathfrak{x}}}}\left(\mathcal{M}, \mathscr{C}_{N \mid X}\right) \longrightarrow \mu_{N} \mathbb{R} \mathscr{H}_{o m_{T_{X}}}\left(\mathcal{M}, \mathscr{B}_{M}\right) \otimes \text { or }_{N \mid M} \text { at } \xi \in T_{N}^{*} M \text {. }
$$

It remains to apply the division theorem in $\mathscr{C}_{N \mid X}$ for micro-differential systems of Kashiwara-Kawai [7].

Corollary 4.11 (local Bochner type extension theorem). Let $\xi \in T_{N}^{*} M$ and $\mathcal{M}$ as in the above theorem. Then there exists an open convex cone $\Omega_{0} \subset M$ with the edge $N$ such that the polar set of $C_{N}\left(\Omega_{0}\right) \subset T_{N} M$ contains $\xi$ in its interior, and every hyperfunction (real analytic) solution $u \in \Gamma_{\Omega} \mathscr{H}_{\text {orn }} \mathscr{\mathscr { C }}_{x}\left(\mathcal{M}, \mathscr{B}_{M}\right)$ on $\Omega:=M \backslash \Omega_{0}$ automatically extends to an open neighborhood of $N$ as a hyperfunction (real analytic) solution to $M$.

We used the micro-hyperbolicity of $M$ on $T_{M}^{*} X$ to show that the extensions of real analytic solutions to $\mathcal{M}$ become also real analytic. These results are slight generalizations of Theorem 3.9 of $[23]$ to the cases where the hyperbolic factors of $\mathcal{M}$ are not necessarily non-characteristic for $Y$. They can be of codimension one in $T^{*} X$. In Theorem 2 of Uchida [27] a result on the extension of "real analytic" solutions is proved in the same situation. However he assumes the smoothness of the characteristic variety on $\dot{T}_{M}^{*} X$. Moreover in his case, every irreducible component of $\mathrm{Ch} \mathcal{M}$ has to be of codimension $\geq 2$.

Remark 4.12. In Corollary 4.11, if we assume in addition the conditions:

(A) The characteristic variety of $\mathcal{M}$ is real and smooth on $\dot{T}_{M}^{*} X$

(B) At each point $p \in T_{M}^{*} X$ the system $\mathcal{M}$ can be transformed to a direct sum of the partial de Rham system (as an $\mathscr{E}_{X}$-module) by a real quantized 
contact transformation,

then the extensions of distribution solutions to $\mathcal{M}$ on $\Omega=M \backslash \Omega_{0}$ are again distributions. That is, we have an isomorphism:

$$
\left.\left.\Gamma_{\Omega} \mathscr{H}_{o m_{\mathscr{D}_{X}}}\left(\mathcal{M}, \mathscr{D} b_{M}\right)\right|_{N} \longrightarrow \mathscr{H}_{o m_{\mathscr{D}_{X}}}\left(\mathcal{M}, \mathscr{D} b_{M}\right)\right|_{N}
$$

This can be proved by the fact that the tempered microfunction solutions $\mathscr{H}_{0 m_{\mathscr{P}_{\lambda}}}$ $\left(\mathcal{M}, \mathscr{C}_{M}^{f}\right)$ to the partial de Rham system are constant along the real bicharacteristic strips of the real part $T_{M}^{*} X \cap \mathrm{Ch} \mathcal{M}$ of Ch $\mathcal{M}$. Also notice the real bicharacteristic strips are transversal to the hypersurface of $M$ defined by $\xi \in$ $T_{N}^{*} M$ in this case.

Example 4.13. Let $Q \in \mathscr{D}_{X}$ be a hyperbolic differential operator in the direction $\xi \in T_{N}^{*} M$. We take elliptic operators $E_{j}(j=1, \cdots, d)$ such that the set $\bigcap_{j=1}^{d}\left\{\sigma\left(E_{\jmath}\right)=0\right\} T^{*} X$ is non-characteristic for $Y$ and set $P_{\jmath}=E_{j} Q+$ (lower order terms). Then for the system $M=\mathscr{D}_{X} / \sum_{j=1}^{d} \mathscr{D}_{X} P$, we have

$$
H^{\prime} \mu_{N} \mathbb{R} \mathscr{H}_{o m_{\mathscr{D}_{X}}}\left(\mathcal{N}, \mathscr{B}_{M}\right) \simeq 0 \text { for } j<d .
$$

and a local Bochner type extension theorem for hyperfunction (real analytic) solutions holds. Note that $\mathscr{D}$-modules $\mathcal{M}$ obtained in this way are not non-characteristic for $Y$ in general.

By adding hyperbolic factors to Theorem 4.5, we have the following theorem.

Theorem 4.14. We suppose that the system $M$ satisfies the conditions:

(i) $M$ is non-characteristic for $Y$.

(ii) $M$ is partially elliptic along $V$ on $N \times{ }_{L} \dot{T}_{L}^{*} X$.

(iii) $M$ is non-microcharacteristic for $Y$ along $V$ on $N \times{ }_{L} \dot{T}_{L}^{*} X$.

(iv) $M$ is micro-hyperbolic in a direction $\xi \in T_{N}^{*} M$ on $N \times{ }_{M} T_{M}^{*} X-N \times{ }_{L} T_{L}^{*} X$.

Then we have for every $j<d$ :

$$
H^{j} \mu_{N} \mathbb{R} \mathscr{H}_{o m_{\mathscr{D}_{X}}}\left(\mathcal{M}, \mathscr{B}_{M}\right)_{\xi} \simeq 0 \text {. }
$$

and a local Bochner type extension theorem for hyperfunction (real analytic) solutions (like Corollary 4.11) holds.

Proof. Set $\dot{\pi}_{M}: T_{N}^{*} M \times_{M} \dot{T}_{M}^{*} X \rightarrow T_{N}^{*} M$. Then we have $H^{\prime} \mathbb{R} \mathscr{H}_{o m_{\mathscr{X}_{X}}}\left(\mathcal{M}, \mathscr{C}_{N M}\right) \simeq 0$ for every $j<d$ on $\pi_{M}^{-1}(\xi)$ by using the preceding results. Thus the conclusion follows from the distinguished triangle in Proposition 4.2.

Remark 4.15. As is clear from the proof of Theorem 3.11 of [23]. Theorem 4.14 is useful also to get an edge of the wedge theorem of Bogoliubov type for hyperfunction (real analytic) solutions. The details are left to the reader. 


\section{$\S 5$. Solvability of Systems with Multiple Characteristics}

In this section, we will make use of the techniques of microlocal inverse image to extend some results of [24] on the solvability of partial differential operators. We employ the notations in the previous sections, but we assume that the codimension d of $N$ in $M$ equals to one. We write $N=\left\{x_{1}=0\right\} \subset M$. First recall the following definition.

Definition 5.1 ([23]). We say that a system $\mathcal{M}$ is "microlocally hyperbolic" in the direction $\xi \in T^{*} M$ if the characteristic variety of $M$ satisfies the condition:

$$
i\left(\dot{\pi}^{-1}(\xi)\right) \cap C_{T_{M X}^{k}}(\operatorname{Ch} M)=\emptyset,
$$

where we have used the natural injection

$$
i: \dot{T}_{M}^{*} X \times_{M} T^{*} M \rightarrow T^{*}\left(T_{M}^{*} X\right) \simeq T_{\left(T_{M}^{*} X^{\prime}\right)}\left(T^{*} X\right)
$$

induced by $\dot{T}_{M}^{*} X \rightarrow M$ and the projection $\dot{\pi}: \dot{T}_{M}^{*} X \times_{M} T^{*} M \rightarrow T^{*} M$.

Example 5.2. Let us take an elliptic operator $E$ (resp. a hyperbolic operator $Q$ in the directions $\pm d x_{1} \in T_{M}^{*} M$ ) on $X$ and set $P=E Q+$ (lower). Then the $\mathscr{D}_{X}$-module $\mathcal{M}=\mathscr{D}_{X} / \mathscr{D}_{X} P$ is not hyperbolic in the directions $\pm d x_{1} \in \dot{T}_{N}^{*} M$ in general but satisfies the condition of microlocal hyperbolicity.

We shall explain how to extend this definition to our relative setting $f: M \rightarrow$ $M^{\prime \prime}$. According to a result of Laurent [14], the normal bundle $T_{V}\left(T^{*} X\right) \simeq T^{*}$ $\left(X / X^{\prime \prime}\right) \times_{X} V$ is endowed with a relative canonical 2-form $\Omega^{r}$. When $X=X^{\prime} \times X^{\prime \prime}$ $=\mathbb{C}_{z^{\prime}}^{l} \times \mathbb{C}_{z^{\prime \prime}}^{n-l}$ and $T^{*}\left(X / X^{\prime \prime}\right) \times_{X} V \simeq T^{*} X^{\prime} \times T^{*} X^{\prime \prime}=\left\{\left(z^{\prime}, z^{\prime \prime} ; \zeta^{\prime} d z^{\prime}+\zeta^{\prime \prime} d z^{\prime \prime}\right)\right\}$, this 2 -form is described by $\sum_{k=1}^{l} d z_{k} \wedge d \zeta_{k}$. Let $\Omega$ be the canonical 2 -form on $T^{*} X$ and take its restriction $\left.\Omega\right|_{V}=\sum_{k=l+1}^{n} d z_{k} \wedge d_{\zeta_{k}}$ to $V$. If we pull back $\left.\Omega\right|_{V}$ by $\tau_{V}$ : $T_{V}\left(T^{*} X\right) \rightarrow V$ and denote it by $\tau_{V}^{*}\left(\left.\Omega\right|_{V}\right)$. then $\Omega^{r}+\tau_{V}^{*}\left(\left.\Omega\right|_{V}\right)$ is a non-degenerated 2 -form on $T_{V}\left(T^{*} X\right)$. Hence we have the Hamiltonian isomorphism :

$$
T\left(T_{V}\left(T^{*} X\right)\right) \longrightarrow T^{*}\left(T_{V}\left(T^{*} X\right)\right)
$$

which induces the isomorphism:

$$
T_{T^{+}\left(M / M^{\prime \prime}\right) \times{ }_{M} \Lambda}\left[T^{*}\left(X / X^{\prime \prime}\right) \times_{X} V\right] \longrightarrow T^{*}\left[T^{*}\left(M / M^{\prime \prime}\right) \times{ }_{M} \Lambda\right]
$$

We also use the injection:

$$
i_{f}:\left[T^{*}\left(M / M^{\prime \prime}\right) \times{ }_{M} \Lambda\right] \times{ }_{M} T^{*} M \longrightarrow T^{*}\left[\left(T^{*}\left(M / M^{\prime \prime}\right) \times{ }_{M} \Lambda\right]\right.
$$

and the projection:

$$
\dot{\pi}_{f}:\left[\dot{T}^{*}\left(M / M^{\prime \prime}\right) \times{ }_{M} \Lambda\right] \times{ }_{M} T^{*} M \rightarrow T^{*} M .
$$


Definition 5.3 . Let $\mathcal{M}$ be a coherent $\mathscr{D}_{X}$-module. We say $\mathcal{M}$ is "microlocally relative hyperbolic" (w.r.t.f) in the direction $\xi \in \dot{T}^{*} M$ if $\mathcal{M}$ satisfies:

$$
i_{f}\left(\dot{\pi}_{f}^{-1}(\xi)\right) \cap C_{\left.T^{*} M / M^{\prime \prime}\right) \times{ }_{M} \Lambda}\left[C_{V}(\mathrm{Ch} M)\right]=\emptyset .
$$

Example 5.4. We assume locally $X \simeq X^{\prime} \times X^{\prime \prime}=\mathbb{C}_{z^{\prime}}^{l} \times \mathbb{C}_{z^{\prime \prime}}^{n-l}$ and $N \simeq N^{\prime} \times M^{\prime \prime}$. Take an elliptic operator $E$ (resp. a hyperbolic operator $Q$ in the directions \pm $d x_{1} \in \dot{T}_{N^{\prime}}^{*} M^{\prime}$ ) on $X^{\prime}$ and set $P=E Q+$ (lower) by taking arbitrary lower order terms from $\mathscr{D}_{X}$. Then the $\mathscr{D}_{X}$-module $\mathcal{M}=\mathscr{D}_{X} / \mathscr{D}_{X} P$ is microlocally relative hyperbolic (w.r.t. $f$ ) in the directions $\pm d x_{1} \in \dot{T}_{N}^{*} M$.

Although these oparators are not partially elliptic nor micro-hyperbolic. we could prove in Theorem 5.2 of [24] the solvability in the sheaf $\mathscr{B}_{M}$ (and in the sheaf $\mathscr{C}_{M}$ of microfunctions) with the aid of the theorem of bimicrolocalization. Note that there exists a complicated intersection of two factors $\{\sigma(E)=0\}$ and $\{\sigma(Q)=0\}$ on the regular involutive submanifold $\Lambda=M \times_{L} T_{L}^{*} X \subset T_{M}^{*} X$. At that time, we treated the differential operators whose symbols contain only the derivations in $z^{\prime}$-variables. We can generalize this result as follows.

Theorem 5.5. Let $\mathcal{M}$ be a coherent $\mathscr{D}_{X}$-module and $p \in \dot{\Lambda}_{N}:=N \times{ }_{L} \dot{T}_{L}^{*} X$. Wc suppose the following conditions:

(i) $\mathcal{M}$ is non-characteristic for $Y$.

(ii) There exists an open conic neighborhood $U$ of $p$ in $T^{*} X$ such that the cut-off $i \cap C h M$ at $p$ of the characteristic variety of $M$ satisfies:

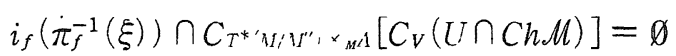

for every $\xi \in \dot{T}_{N}^{*} M$.

(iii) $M$ is non-microcharacteristic for $Y$ along $V$ at $p \in \dot{\Lambda}_{N}$.

Then we have the vanishing of cohomologies:

$$
H^{J} \mathbb{R} \mathscr{H}_{o r n}\left(\mathcal{M}, \mathscr{C}_{M}\right) \simeq 0 \text { for } j>\text { proj.dim } \mathcal{M}_{Y}
$$

at $p \in \dot{\Lambda}_{N}$

Proof. Set $\dot{\pi}_{L}: \dot{T}_{M}^{*} L \times_{L} T_{L}^{*} X \rightarrow \Lambda$ and consider the sheaf $\mathscr{C}_{M L}:=\mu_{M L}\left(\mathscr{O}_{X}\right) \otimes$ or $_{M}$ [n] on $T_{M}^{*} L \times{ }_{L} T_{L}^{*} X$ introduced by Kataoka-Tose [13] and [19]. Then we have the distinguished triangle on $\Lambda$ :

$$
\text { (5.9) }\left.\left.\mathbb{R} \mathscr{H}_{o m_{\mathscr{C}_{x}}}(\mathcal{M}, \mathscr{C O})\right|_{\Lambda} \rightarrow \mathbb{R} \mathscr{H}_{o m_{\mathscr{D}_{x}}}\left(\mathcal{M}, \mathscr{C}_{M}\right)\right|_{\Lambda} \rightarrow \mathbb{R} \pi_{L}, \mathbb{R} \mathscr{H}_{o m_{\mathscr{T}_{x}}}\left(\mathcal{M}, \mathscr{C}_{M L}\right) \longrightarrow+1 \text {, }
$$

where $\mathscr{C} \mathscr{O}=\mu_{L}\left(\mathscr{O}_{X}\right) \otimes$ or $_{L}[n-l]$ denotes the sheaf of microfunctions with holomorphic parameters. We also consider the sheaf $\mathscr{C} \mathscr{O}^{\prime}=\mu_{H}\left(\mathscr{O}_{Y}\right) \otimes \operatorname{or}_{H}[n-l]$ on $T_{H}^{*} Y$ and identify $\Lambda_{N}$ and $N \times{ }_{H} T_{H}^{*} Y$ via the projection $N \times{ }_{M} T_{M}^{*} X \rightarrow T_{N}^{*} Y$. By Schapira's Cauchy-Kowalevski type theorem in [18] and the assumption (ni), 
there is an isomorphism

$$
\mathbb{R} \mathscr{H}_{o m_{\mathscr{D}_{\boldsymbol{X}}}}(\mathcal{M}, \mathscr{C} \mathfrak{O}) \stackrel{\sim}{\longrightarrow} \mathbb{R} \mathscr{H}_{o m_{\mathscr{F}_{Y}}}\left(._{\mathscr{U}}^{U}, \mathscr{C} \mathscr{O}^{\prime}\right)
$$

on $\Lambda_{N}$ for a sufficiently small conic open neighborhood $U$ of $p$ in $T^{*} X$, where $\mathcal{M}_{Y}^{U}$ stands for the induced system of $\left.\mathcal{M}\right|_{U}$ to $Y$ in the category of $\mathscr{E}_{X}$-modules. Therefore the first term of (5.9) is a direct summand of the complex $R \mathscr{H}_{O m_{D_{r}}}$ $\left(\mathcal{M}_{Y}, \mathscr{C} \mathscr{O}^{\prime}\right)$ and

$$
H^{\prime} \mathbf{R} \mathscr{H}_{o m_{D_{x}}}(\mathcal{M}, \mathscr{C O})_{p} \simeq 0 \text { for } j>\text { proj.dim } M_{Y}
$$

holds. Next we shall use the natural embedings:

$$
T_{N}^{*} L \times{ }_{L} T_{L}^{*} X \stackrel{\delta}{ }\left(N \times{ }_{M} T_{M}^{*} L\right) \times{ }_{L} T_{L}^{*} X \stackrel{\varpi}{\longrightarrow} T_{M}^{*} L \times{ }_{L} T_{L}^{*} X
$$

and the projection $\pi_{N}: T_{N}^{*} L \times{ }_{L} T_{L}^{*} X \longrightarrow \Lambda_{N}$. Then it follows from the proof of Theorem 5.1 of [24] and the assumption (ii) that the canonical morphism:

$$
\delta_{*} \varpi^{-1} \mathbb{R} \mathscr{H}_{o m_{\mathscr{D}_{X}}}\left(\mathcal{M}, \mathscr{C}_{M L}\right) \simeq \mathbb{R} \mathscr{H}_{o m_{\mathscr{D}_{x}}}\left(\mathcal{M}, \mathscr{C}_{N L}\right)[1]
$$

is an isomorphism on $\pi_{N}^{-1}(p) \cap\left(N \times{ }_{M} \dot{T}_{M}^{*} L\right) \times{ }_{L} T_{L}^{*} X$. Hence the third term of $(5.9)$ is a direct summand of the complex $\mathbf{R} \dot{\pi} * \mathbf{R} \mathscr{H}_{o m_{D_{X}}}\left(\mathcal{M}, \mathscr{C}_{N L}\right)[1]$ for $\dot{\pi}: \dot{T}_{N}^{*} L$ $\times_{L} T_{L}^{*} X \longrightarrow \Lambda_{N}$. Now recall the chain of isomorphisms in (3.22) obtained by the microlocal inverse image $h^{-1} F_{0}(h: Y \rightarrow X)$ and set $\dot{\pi}_{H}: \dot{T}_{N}^{*} H \times_{H} T_{H}^{*} Y \longrightarrow \Lambda_{N}$. Then we have the isomorphism:

$$
\mathbb{R} \rho, \mathbb{R} \mathscr{H}_{o m_{\mathscr{L}_{\lambda}}}\left(\mathcal{M}, \mathscr{C}_{N L}\right)[1] \simeq \mu_{N H}\left(h^{-1} F_{0}\right) \otimes \operatorname{or}_{N}[n-1]
$$

on $\dot{\pi}_{H}^{-1}(p)$ and the complex $\mathbb{R} \pi * \mathbb{R} \mathscr{H}_{o m_{\mathscr{D}_{x}}}\left(\mathcal{M}, \mathscr{C}_{N L}\right)_{p}[1]$ is also a direct summand of $\mathbb{R} \pi_{H_{+}} \mathbb{R} \mathscr{H} o m_{\mathscr{D}_{Y}}\left(\mathcal{M}_{Y}, \mathscr{C}_{N H}\right)_{p}$. Finally use the biconically flabbiness of the sheaf $\mathscr{C}_{N H}$ proved by [13] to show the vanishing:

$$
H^{\prime} \mathbf{R} \dot{\pi}_{H}, \mathbb{R} \mathscr{H}_{o m_{\mathscr{D}_{Y}}}\left(\mathcal{M}_{Y}, \mathscr{C}_{N H}\right)_{p} \simeq 0 \text { for } j>\text { proj.dim } \mathcal{M}_{Y} .
$$

It completes the proof of the theorem.

Our arguments in the proof above is also useful to prove the solvability in the sheaf $\mathscr{B}_{M}$ of hyperfunctions. In the course of the proof of the following corollary (example), we use Theorem 3.1 of Kataoka-Tose [12] as well as the proof of Theorem 5.2 and Theorem 5.5 of [24]. We assume locally $X \simeq X^{\prime} \times X^{\prime \prime}$.

Corollary 5.6. Let $E \in \mathscr{D}_{X^{\prime}}$ (resp. $E_{0} \in \mathscr{D}_{X}$ ) be an elliptic operator on $X^{\prime}$ (resp. on $X)$ and $Q \in \mathscr{D}_{X^{\prime}}\left(\right.$ resp. $\left.Q_{0} \in \mathscr{D}_{X}\right)$ a hyperbolic operator in $\pm d x_{1}$-directions on $X^{\prime}$ (resp. on $X)$. We set $P=E E_{0} Q Q_{0}+$ (lower) by taking arbitrary lower order terms from $\mathscr{D}_{X}$. We also assume the separation condition:

$$
M \times{ }_{L} \dot{T}_{L}^{*} X \cap\left\{\sigma\left(Q_{0}\right)=0\right\}=\emptyset
$$

Then

(i) $P: \mathscr{B}_{M} \longrightarrow \mathscr{B}_{M}$ is surjective. 
(ii) $P: \mathscr{C}_{M} \longrightarrow \mathscr{C}_{M}$ is surjective at any point of $\dot{T}_{M}^{*} X$.

Remark 5.7. Corollary 5.6 extends Theorem 5.2 and Theorem 5.5 of [24]. Note that Theorem 5.2 of [24] was a natural generalization of the $\mathscr{C}_{M^{-}}$-solvability for partially elliptic operators due to Bony-Schapira [2].

\section{References}

[1] Bony, J-M., Extension du théorème de Holmgren. Sém. Goulaoulc-Schwartz. 1975-76, exposé 17.

[2] Bony. J-M. and Schapira. P.. Propagation des sıngularités analytıques pour les solutions des équations aux dérivées partielles, Ann. Inst. Fourier. Grenoble, 26, I (1976), 81-140.

[3] D'Agnolo, A., Inverse image for the functor $\mu$ hom, Publ. RIMS, Kyoto Unv.. 27 (1991), 509-532.

[4] D'Agnolo, A. and Schapira. P.. An inverse 1mage theorem for sheaves with applications to the Cauchy problem, Duke Math. J., 64 (1991), 451-472.

[5] Delort, J-M., Microlocalisation simultanée et problème de Cauchy ramifié, Compositıo Math., 100 (1996). 171-204.

[6] Funakoshi, S.. Master thesis presented to the University of Tokyo (1995).

[7] Kashıwara. M. and Kawaı, T., On the boundary value problem for elliptic systenıs of linear partial differential equations I - II. Proc. Japan. Acad., 48 (1971), 712-715; ibid., 49 (1972). 164-168.

[8] Kashiwara. M. and Laurent, Y., Théorèmes d'annulation et deuxième microlocalisation. prépublıcation d'Orsay. 1983.

[9] Kashiwara, M. and Schapira, P., Micro-hyperbolic systems, Acta Math., 142 (1979), 1-55.

[10] Sheaves on manifolds, Grundlehlen Math. Wiss., 292, Springer-Verlag(1990).

[11] Kataoka, K., Microlocal theory of boundary value problems I - II, J. Fac. Sci. Liniv. Tokyo, 27 (1980), 355-399; ıbıd., 28(1981), 31-56.

[12] Kataoka, K. and Tose, N., Vanıshing theorems for the sheaf of microfunctions with holomorphic parameters, J. Fac. Sct. Univi. Tokyo, 35 (1988). 313-320.

[13] - Some remarks in 2nd microlocalızation (in Japanese), RIMS Kôkyu-roku, Kyoto Unvv., 660 (1988), 52-63.

[14] Laurent, Y., Theone de la deuxiéme microlocalisation dans le domaine complexe. Progr. Math., 53. Birkhauser, Boston (1985).

[15] Martineau. A., Le 'edge of the wedge theorem' en théorie des hyperfonctions de Sato, Proc. Intern. Conf. on Functional Analysis and Related Topics, 1969. Univ. Tokyo Press, Tokyo (1970). 95-106.

[16] Morımoto, M., Sur la décomposition du faisceau des germes de singularités d'hyperfonctions, J. Fac. Sci. Univ. Tokyo. 17 (1970), 215-239.

[17] Sato. M., Kawai, T. and Kashıwara. M., Hyperfunctions and pseudodifferentıal equations, L.N. in Math, 287. Springer-Verlag (1973), 265-529.

[18] Schapira, P., Propagation at the Boundary of Analytic singularities, in Singularities of Boundary Value Problems, Reidel Publ. Co. (1981), 185-212.

[19] Schapira, P. and Takeuchi, K.. Déformation binormale et bispécialisation, C.R. Acad. Sc.. 319. Série I (1994), 707-712.

[20] Schapira. P. and Trépreau, J-M., Microlocal pseudoconvexity and edge-of-the-wedge theorem. Duke Math. J., 61 (1990), 105-118. 
[21] Takeuchi, K., Binormal deformation and bimicrolocalization, Publ. RIMS, Kyoto Univ., 32 (1996), 277-322.

[22] - Théorèmes de type Edge of the Wedge pour les solutions hyperfonctions, C.R. Acad. Sc., 321. Série I (1995), 1333-1336.

[23] - Edge-of-the-wedge type theorems for hyperfunction solutions, Duke Math. J, 89 (1997), 109-132.

[24] Takeuchi, K. and Koshimizu, H., A method of desingulanzution for the study of operators with multiple characteristics, submittıng.

[25] Tonin, F.. The functor of moderate cohomology and the Cauchy problem, preprint (1995).

[26] Tose. N., Theory of partially ell lptic systems and its applications, Master thesis presented to the University of Tokyo (1985).

[27] Uchida, M., Continuation of analytic solutions of linear differential equations up to convex conical sungularities, Bull. Soc Math. France, 121 (1993), 133-152.

[28] A generalization of Bochner's tube theorem for elliptic boundary value problems. RIMS Kôkyu-roku. Kyoto Univ.. 845 (1993), 129-138. 
